\title{
Immunocompromised and immunocompetent mouse models for head and neck squamous cell carcinoma
}

\author{
Zhen-ge Leil,* \\ Xiao-hua $\operatorname{Ren}^{2, *}$ \\ Sha-sha Wang ${ }^{3}$ \\ Xin-hua Liang ${ }^{3,4}$ \\ Ya-ling Tang 3 \\ 'Department of Oral and Maxillofacial \\ Surgery, Stomatological Hospital \\ Affiliated to Nanchang University, \\ Nanchang, Jiangxi, ${ }^{2}$ Department \\ of Stomatology, Sichuan Medical \\ Science Academy and Sichuan \\ Provincial People's Hospital, ${ }^{3}$ State \\ Key Laboratory of Oral Diseases, \\ West China Hospital of Stomatology, \\ Sichuan University, ${ }^{4}$ Department \\ of Oral and Maxillofacial Surgery, \\ West China College of Stomatology, \\ Sichuan University, ${ }^{5}$ Department of \\ Oral Pathology, West China Hospital \\ of Stomatology, Sichuan University, \\ Chengdu, Sichuan, People's Republic \\ of China \\ *These authors contributed equally \\ to this work
}

Correspondence: Ya-ling Tang State Key Laboratory of Oral Diseases, West China Hospital of Stomatology, Sichuan University, No I4, Section 3, Renminnan Road, Chengdu 61004I, Sichuan, People's Republic of China Tel +86 I50 08458098

Email tangyaling@scu.edu.cn

Xin-hua Liang

Department of Oral and Maxillofacial Surgery, West China College of Stomatology, Sichuan University, No 14, Section 3, Renminnan Road, Chengdu 61004I, Sichuan, People's Republic of China Tel +86 I58 84483965

Email Ixh88866@scu.edu.cn
This article was published in the following Dove Press journal:

OncoTargets and Therapy

27 January 2016

Number of times this article has been viewed

Abstract: Mouse models can closely mimic human oral squamous epithelial carcinogenesis, greatly expand the in vivo research possibilities, and play a critical role in the development of diagnosis, monitoring, and treatment of head and neck squamous cell carcinoma. With the development of the recent research on the contribution of immunity/inflammation to cancer initiation and progression, mouse models have been divided into two categories, namely, immunocompromised and immunocompetent mouse models. And thus, this paper will review these two kinds of models applied in head and neck squamous cell carcinoma to provide a platform to understand the complicated histological, molecular, and genetic changes of oral squamous epithelial tumorigenesis.

Keywords: head and neck squamous cell carcinoma, HNSCC, mouse models, immunocompromised models, immunocompetent models, transgenic models

\section{Introduction}

Head and neck squamous cell carcinoma (HNSCC) is the sixth most prevalent malignancy in the world and is the third most common cancer in developing countries. ${ }^{1,2}$ HNSCC affects the nasal cavity and paranasal sinuses, the nasopharynx, oropharynx, and the oral cavity. Oral squamous cell carcinoma (OSCC) develops from the epithelium of the oral cavity, including tongue, lips, gums, floor of the mouth, cheeks, hard palate, or other unspecified parts of the mouth. ${ }^{3}$ Despite the significant advances made in conventional treatment such as surgery, radiotherapy, chemotherapy, or combinations of these over the past decade, HNSCC continues to have a dismal prognosis, with a 5 -year overall and disease-free survival of estimated $\sim 50 \%{ }^{4}$ Thus, the complicated histological, molecular, and genetic changes of oral and pharynx squamous epithelial tumorigenesis are needed to be thoroughly understood to provide a new therapeutic opportunity and better prognosis for HNSCC patients.

Experimental animal models, which accurately represent the cellular and molecular changes associated with the initiation and progression of human cancer, are thus of crucial importance in the field of cancer research. ${ }^{5,6}$ Mice are small in size, inexpensive to maintain, reproduce rapidly and genetic engineering can be performed easily compared to other animals. ${ }^{7}$ Mouse models can closely mimic human oral squamous epithelial carcinogenesis, greatly expand the in vivo research possibilities, and play a critical role in investigating the initiation and development of HNSCC and testing new therapeutic approaches. ${ }^{8}$ With the development of transgenic technology and emergence of in vivo imaging technology, mouse models could more and more closely simulate the progression of HNSCC than ever before. ${ }^{9,10}$ 


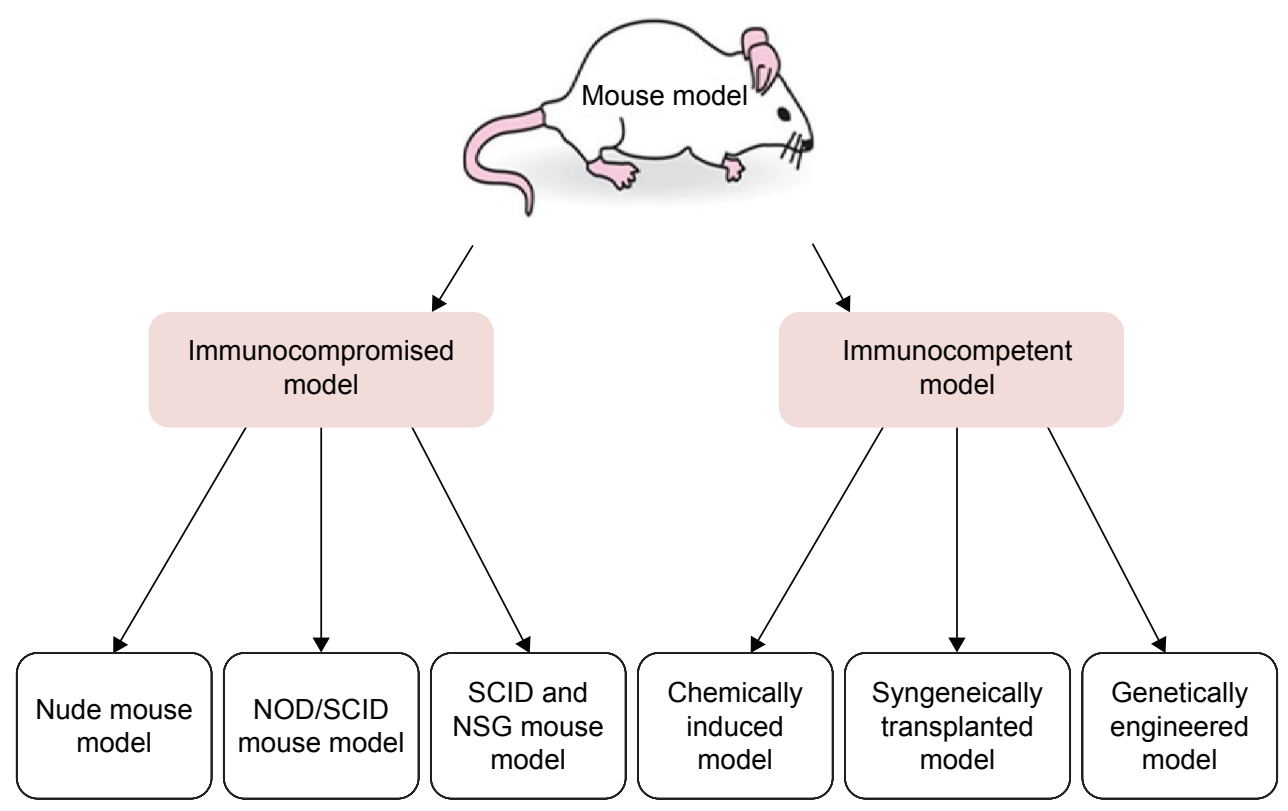

Figure I Mouse models of oral cancer.

Abbreviations: NOD, nonobese diabetic; SCID, severe combined immunodeficiency; NSG, NOD/SCID gamma.

Although the molecular mechanisms involved in the association of immunity/inflammation and cancer have remained elusive at present, on the basis of facts that patients with chronic inflammation diseases have a substantially increased risk of developing cancer and that inflammatory cells and immune factors were present in biopsied tumor samples, the role of immunity/inflammation in tumorigenesis has been generally addressed, and the increasing evidence showed that inflammatory/inflammation microenvironment was an essential component of tumors..$^{2,1-14}$ Hanahan and Weinberg ${ }^{15}$ have proposed that six hallmarks of cancer together constitute an organizing principle that provides a logical framework for understanding the remarkable diversity of neoplastic diseases in 2000. With the conceptual progression in the following 10 years, two emerging hallmarks, reprogramming energy metabolism and evading immune destruction, were added to the cancer hallmarks list in $2010 .{ }^{16}$ This strongly highlighted the role of immunity in cancer. Thus, based on the immune state of cancer, this review summarizes immunocompromised and immunocompetent mouse models applied in the research of HNSCC to contribute to our understanding of HNSCC biology (Figure 1).

\section{Immunocompromised model}

In 1960s, a mutation of thymus dysgenesis in mice was discovered, and these mice were called nude mice. ${ }^{17,18}$ Rygaard and Povlsen ${ }^{19}$ originally established a human transplanted sigmoid colon adenocarcinoma model by subcutaneously injecting tumor cell suspension into nude mice. In 1983, severe combined immunodeficiency (SCID) mice were reported, which were more immunodeficient than nude mice. ${ }^{20}$ In SCID mice, there are defects in T- and B-lymphocytes, but the function of natural killer (NK) cells and macrophages is normal. In 1992, an extremely immunodeficient strain of mice was reported by the Jackson Laboratory, which was called nonobese diabetic/SCID (NOD/SCID) mouse. ${ }^{21}$ NOD/SCID mice not only lacked functional lymphoid cells similar to SCID mice, but also showed reduced activation of NK cells and had less mature macrophage population..$^{22}$

All these mice were defined as immunocompromised models or xenograft models, which were used to transplant heterologous cells or tissues to establish models systems. To date, these models have been extensively applied in the research of the molecular mechanism of antitumor effect of chemicals on HNSCC, combination of therapies, invasion and metastasis, epithelial-mesenchymal transition, and cancer stem cells (CSCs), especially when in vitro results need to be validated in vivo.

Povlsen and Rygaard ${ }^{23}$ first reported that three out of four human epidermoid carcinomas at head and neck region were successfully transplanted into nude mice consecutively. Nude mice model for HNSCC research was discussed as early as $1984^{24}$ and had become the most widely immunocompromised model to be applied in HNSCC. Nude mice transplanted models were used to confirm the inhibitory function of some medicine or factors during tumor development 
in clinical trials, such as Safrole, BRAK/CXCL14, and doxycycline, which helped to identify the inhibition effect on the growth of HNSCC in vitro. ${ }^{25-27}$ Using a xenograft model of nude mice, our group found that local hyperthermia acted in an antilymphangiogenic role by suppressing the expression of tumor VEGF-C and VEGF-D, and thereby inhibiting cancer cell lymphatic metastasis in tongue SCC. ${ }^{28}$ And, we observed that knocking down HIF-1 $\alpha$ or HIF-2 $\alpha$ individually inhibited the xenograft tumor angiogenesis and growth, and knocking them down simultaneously revealed a better inhibitory effect than knocking down either unit alone. ${ }^{29}$ In addition, several combinations of therapies, such as lentinan in combination with fluoropyrimidine, radiotherapy in combination with cetuximab, tirapazamine, and MnSOD-plasmid liposome gene therapy, and rapamycin in combination with cisplatin treatment, were shown to be potentially improved strategies for HNSCC in nude mice, ${ }^{30-32}$ which has identified the role and prospect of the nude mice in studying antitumor effect of chemicals and combination of therapies in HNSCC.

Understanding the molecular mechanisms in the invasion and metastasis of HNSCC is urgently needed for new therapeutic intervention. In 1990, the first mouse model system for metastasis of HNSCC was established by implanting human laryngeal SCC cell lines into the floor of the mouth in nude mice. Pulmonary metastases were noted in $44 \%$, bone invasion in $80 \%$, angioinvasion in $76 \%$, and soft tissue invasion in $96 \%$ of the animals. ${ }^{33}$ Subsequently, various methods were used to build HNSCC mouse models that were prone to metastasis. In 1995, Kawashiri et $\mathrm{al}^{34}$ found that, comparing with the back tumors of nude mice, orthotopic (tongue or the oral floor) tumor exhibited analogous invasive growth and the histological appearance to the original tumors. In this model with OSC-19 cell lines, metastasis to the cervical lymph node was markedly inhibited by cisplatin or peplomycin and the combination of neoadjuvant chemotherapy and tumor resection. ${ }^{35,36}$ Another experimental model of invasion and metastasis was built by orthotopic transplantation of HSC-2, HSC-3, and HSC-3 cell lines into tongue of nude mice. ${ }^{37}$

Bone invasion attracted great attention as a common behavior of HNSCC, which always caused facial defects and dysfunction of mandibular and maxillary bone and affected the prognosis. By injecting UM-SCC-1 cell lines into mylohyoid muscle of nude mice, the investigator got a model which allowed quantification of tumor invasion. ${ }^{38} \mathrm{In}$ order to make another bone invasion model, human OSCC cell lines (SCC14a, SCC1, and SCC12) were injected into the surface of calvaria in $\mathrm{NCr}-\mathrm{nu} / \mathrm{nu}$ athymic mice. A higher tumor growth and increased rate of osteolysis were found in SCC14a tumor bearing mice, which was related to high level production of CXCL13. ${ }^{39}$ These mouse models with metastasis aimed to elucidate the molecular mechanism of the invasion and metastasis in HNSCC.

Yadav et al, ${ }^{40}$ through transplanting head and neck tumor cell line (CAL27) into SCID mice, showed that interleukin-6 (IL-6) was a promoter of HNSCC tumor metastasis and worked by inducing epithelial-mesenchymal transition via the JAK-STAT3-SNAIL signaling pathway. And Shinriki et $\mathrm{al}^{41}$ investigated the inhibitory effects of anti-IL-6 receptor antibody tocilizumab on OSCC growth using SCID mice xenograft model based on in vitro data. In another experiment, Jung et $\mathrm{al}^{42}$ found keratinization-associated miR-7 and miR-21 deregulated tumor suppressor reversion-inducing cysteine-rich protein with kazal motifs in oral cancer which led to aggressiveness of tumors with CAL27 orthotopic xenograft mouse model. Yang et $\mathrm{al}^{43}$ inoculated connective tissue growth factor (CTGF)-overexpressed SAS cells (SAS/ CTGF-M3) into buccal mucosa of SCID mice to establish OSCC model, showing reverse correlation between CTGF and miR-504, miR-504 and FOXP1, and a positive correlation between CTGF and FOXP1. Taken together, the SCID mouse model provides an avenue to analyze OSCCassociated mediators or study it as a potential therapeutic target for OSCC.

Although NOD/SCID mice model was used in research on hematopoietic and lymphatic system at the beginning, it was gradually employed for studying solid tumors. With bioluminescent imaging technology, Lin et $\mathrm{al}^{44}$ injected human transfected SAS/luc cells into NOD/SCID mice, established an OSCC model, and observed a significant inhibition effect of curcumin on tumor growth. Patel et $\mathrm{al}^{45}$ found that rapamycin and the rapalog RAD001 diminished lymphangiogenesis in the primary tumors and prevented the dissemination of HNSCC cells to the cervical lymph nodes, thereby prolonging NOD/SCID mice survival, suggesting that mTOR was a potential target of HNSCC. Recently, the NOD/SCID mouse model has been regarded as the gold standard assay for defining CSCs fraction. ${ }^{46,47}$ With a NOD/ SCID mouse model, Prince et $\mathrm{al}^{48}$ tested the tumorigenic potential of different populations of cancer cells derived from primary, unmanipulated human HNSCC samples and found that the $\mathrm{CD} 44^{+}$population possessed the unique properties of self-renewal and differentiation. Clay et $\mathrm{al}^{49}$ implanted high aldehyde dehydrogenase $\left(\mathrm{ALDH}^{\text {high }}\right)$ activity and $\mathrm{ALDH}^{\text {low }}$ populations of HNSCC cells into NOD/SCID mice and confirmed that ALDH activity was a highly selective 
CSCs marker for HNSCC. In a NOD/SCID xenograft model derived from clinical HNSCC specimens, Sun and Wang ${ }^{50}$ injected sorted cells subcutaneously and found that c-Met ${ }^{+}$ cells were capable of self-renewal and generating tumors and that isolation of HNSCC cells using a second marker CD44 could further enhance the in vivo tumorigenicity.

Recently, NOD SCID gamma (NSG) or NOD/SCIDIL$2 \mathrm{r} \gamma^{\text {null }}$ (NOG) mice have been established, and SCID mice are now subdivided into the NOD/SCID mice and NOG mice. The NOG mouse model combines the features of SCID and IL-2 receptor gamma chain deficiency. Analogously to the NOD/SCID mice, these mice lack mature T-cells, B-cells, and macrophages. For the detection of common cytokine receptor, $\gamma$-chain, this model, which is characterized by extremely low number of NK cells and dysfunctional dendritic cells, is used. As a result, NOG mice had no functional lymphocytes and efficacious NK activity. Hence, NOG mice with most severe degree in the deficiency of immunity are characterized by high xenograftment efficiency, even only a few cells and almost no rejection of human cells and tissues. Although extremely immunodeficient, NOG mice can live for more than $1 \frac{1}{2}$ years under strict specific pathogen-free condition. Compared to NOD/SCID mice, the "leakiness" of T- and B-cells does not occur in NOG mice, though the reason remains to be elucidated. Currently, NOG mice have been used to examine hematopoietic stem cells (HSCs), identify CSCs, and research the embryonic stem cells and induced pluripotent stem cells. These mice also have been applied as models to study cancers and infectious diseases such as HIV-1 infection. Besides, NOG mice can be a used as a platform to develop a new humanized mice model. Shrivastava et $\mathrm{al}^{51}$ established tumor xenografts by direct injection of cells into the flanks of NSG mice to examine the ability to form tumor from parental and oralspheres of OSC19 cells in NSG mice. The result showed that oralspheres contributed to tumor growth and vascularization, indicating that oralspheres might be future therapeutic modalities against treatment resistant HNSCC. ${ }^{51}$

Although the immunocompromised mouse models preventing host rejection were widely applied in HNSCC studies because of their certainty of tumor initiation, speed of tumor development, and easy establishment (injecting cells or transplant tissues subcutaneously or orthotopically in animal), there are still some disadvantages of immunocompromised models. The rate of invasion and metastasis is very uncertain and different, changing from very low to markedly high due to dissimilar transplantation methods, hosts, and transplantation site, though invasion or metastasis was observed in most cases..$^{33,34,37}$ One hundred and six malignant human tumor cell lines including 11 different tumor cell lines were studied in 1,045 nude mice, with only $14(1.3 \%)$ instances of metastasis. ${ }^{52}$ In addition, some studies have showed that many compounds with significant antitumor effects in immunocompromised models do not work in human beings. ${ }^{53}$ Due to the totally different tumor immune microenvironment in immunodeficient mice compared with humans, it is difficult to evaluate the interaction between treatment and immune system by using this kind of model. In a word, the inability to simulate the in vivo inflammation/ immunity environment could be the Achilles' heel of immunocompromised mouse models for HNSCC.

\section{Immunocompetent models Spontaneous models}

The development of immunodeficient mice provides the possibility of establishing humanized mice (mouse models with humanized immune systems, which carry partial or complete human physiological systems), representing ground-breaking platforms to evaluate compounds to treat cancer. ${ }^{54}$ Immunodeficient mice were engrafted with human HSCs or human peripheral blood mononuclear cells, and exogenous administrations of human hormones, growth factors, and cytokines were all needed. Humanized $\mathrm{CD} 34^{+}$mice were produced by injecting $\mathrm{CD}_{3} 4^{+}$and yielded robust multilineage immune systems with good T-cell maturation and function for longterm studies. This model has the longest research span, over 12 months with a functional human immune system, and displays T-cell dependent inflammatory responses, with no donor cell immune reactivity toward host. ${ }^{55,56}$ However, the application of humanized CD $34^{+}$mice in HNSCC has not been reported till now and should be gradually employed in the future.

Humanized mice, one kind of spontaneous HNSCC model, is extremely similar to human tumors and has a high value in studying effects of environmental factors, carcinogenic factors, and genetic factors in tumorigenesis, especially in the immunocompetent environment. However, many animals and much time are needed to establish this kind of model, and it is difficult to reproduce this model. ${ }^{57}$ Due to these limitations, spontaneous HNSCC model can hardly be employed widely.

\section{Chemically induced models}

Initially, inducing oral malignant tumors chemically always failed, because the oral mucosa was more resistant to the action of chemicals than skin. ${ }^{58}$ The most common explanations 
for the difference in susceptibility were sebaceous glands and hair follicles in the skin, and the influence of saliva in the mouth. ${ }^{59}$ Finally, HNSCC was successfully induced in hamster cheek pouch as an animal model, using 9, 10 dimethyl-1, 2, benzanthracene (DMBA), by Salley. ${ }^{60}$ In 1961, Morris ${ }^{61}$ standardized this experimental model. In general, histologically, the carcinogenesis of mucosa was divided into four successive stages: hyperplasia, atypical hyperplasia, carcinoma in situ, and squamous cell carcinoma. However, there was difficulty in distinguishing between the changes of the epithelium caused by direct contact with the carcinogen and true premalignant transformation because they were transient and reversible in the DMBA-induced cheek tumors. DMBA-induced tumors did not possess many of the histological features of differentiated SCC and did not closely resemble early human lesions. ${ }^{62,63}$ More importantly, the tumor occurred in the hamster cheek pouch, which was an immunodeficient area and is absent in human, so this model did not mimic human OSCC very well.

Although DMBA was widely employed in hamster and rat oral cancer models, ${ }^{60,61,64}$ it proved to be difficult to induce oral carcinoma with DMBA in mice models. ${ }^{65}$ 4-Nitroquinoline 1-oxide (4-NQO), a water-soluble quinolone derivative, was then introduced to OSCC model. Administration of 4-NQO with drinking water or a topical application produced a spectrum of carcinogenesis progression that demonstrated multiple dysplastic, preneoplastic, and neoplastic lesions after long-term treatment in both rat and mouse models, and these could mimic human oral cavity neoplastic transformation. ${ }^{66-72} 4-\mathrm{NQO}$-induced mouse model of oral cancer has been gradually adopted and improved. In early research, 4-NQO was painted on the surface of oral mucosa, but this method was laborious and difficult in terms of applying the same dose from one mouse to another. ${ }^{66}$ Furthermore, the progress of carcinogenesis was not as natural as in human. Drinking water administration method was then introduced. This model and method was standardized by Tang et al. ${ }^{67}$ In Tang et al's study, ${ }^{67}$ 4-NQO painted tongues or delivery of 4-NQO in the drinking water were compared in both CBA mice and C57BL/6 mice, and the latter one was found with a much higher incidence of oral cavity carcinogenesis. The concentration of 4-NQO in drinking water $(100 \mu \mathrm{g} / \mathrm{mL})$ and 16 weeks of treatment have been widely adopted in other research that followed.

Many features of human oral cancer development have been reflected in the 4-NQO mice model. With this model, the molecular mechanisms involved in the initiation and progression of HNSCC can be thoroughly comprehended. A research about epithelial-mesenchymal interactions indicated that the underlying connective tissue adjacent to the epithelium was also affected in the later stages of carcinogenesis. ${ }^{73}$ DNA hypomethylation was shown to suppress oral squamous cell carcinogenesis in 4-NQO mice model. ${ }^{74,75}$ It was reported that angiogenesis inhibited by ABT-510 could slow or delay the development of HNSCC. ${ }^{76}$ Inactivation of VEGFR or EGFR could significantly suppress oral cancer development and progression in 4-NQO mouse model. ${ }^{77-79}$ These results could be the clues that would help to find therapeutic target for HNSCC.

\section{Syngeneically transplanted models}

Traditionally, human tumor xenograft models were established by injecting tumor cell lines or transplanting tissues from patients into immunocompromised mouse. But this model was not suitable to dissect the role of tumor and host immunity factor in malignant transformation and metastatic tumor progression of OSCC. Syngeneic transplanted model was achieved by injecting homologous cell lines into immunocompetent mice, with the advantages of easily forming tumor and avoiding the host rejection in immunocompetent environment. ${ }^{80}$

In 1984, with injecting SCCVII/SF cells cultured in vitro subcutaneously into syngeneic $\mathrm{C} 3 \mathrm{H} / \mathrm{HeJ}$ mice, a syngeneic transplanted model was created. Subsequently, positive effects were obtained in the study that combined continuous low dose rate irradiation and concurrent infusion of bleomycin, cyclophosphamide, cis-platinum, 5-fluorouracil, actinomycin D, and mitomycin C, which is chemoradiotherapy. ${ }^{81}$ By injecting the same cell line into the floor of the mouth of $\mathrm{C} 3 \mathrm{H} / \mathrm{HeJ}$ mice, O'Malley et $\mathrm{al}^{82}$ established an OSCC model and presented local invasion and pulmonary metastases. Another oral cancer model was built by implanting AT-84 (a spontaneously arising OSCC of $\mathrm{C} 3 \mathrm{H}$ mice) cell line into syngeneic mice to study the mechanisms involved in immune-mediated therapy of HNSCC. ${ }^{83}$ Cell line derived from 4-NQO-mediated tumorigenic SCID mice was inoculated into $\mathrm{BALB} / \mathrm{c}$ and congenic SCID mice to determine whether CD80 expression affected the immune response and therapy in OSCC. ${ }^{84}$ In this syngeneic mouse model, the combined use of IL-12 and IL-2 resulted in significantly greater tumor reduction than with either one alone. ${ }^{85}$ In Meth-A fibrosarcoma-bearing BALB/c mice or SCCVII-bearing $\mathrm{C} 3 \mathrm{H} / \mathrm{HeN}$ mice, local dendritic cell therapy with concurrent administration of TS-1 and OK-432 was a useful strategy for the treatment of solid tumors. And the potential mechanism was related to TLR4 signaling. ${ }^{86}$ 
Within the immunocompetent environment, syngeneic transplanted models possess better application prospects for investigating the mechanism of OSCC invasion and metastasis. Cui et al and Nomura et al ${ }^{87,88}$ inoculated SCCVII cells into masseter region of $\mathrm{C} 3 \mathrm{H} / \mathrm{HeN}$ mice to establish a bone invasion model. They found that various cancerderived cytokines, such as IL-6, PTHrP, TNF- $\alpha$, RANK, RANKL, osteoproteger, played important roles in the OSCC invading bone, and by inhibiting these cytokines, YM529 (a third-generation bisphosphonate) could suppress osteoclastmediated bone invasion. A similar model was also used by Lee et al ${ }^{89}$ and Furuta et al. ${ }^{90}$ In another research, MDA1986 cell lines were inoculated into tongue orthotopically; however, the incidence of regional and systemic metastases was too low. To increase cell line metastatic potential, Myers et $\mathrm{a}^{11}$ isolated tumor cells from cervical lymph nodes and performed serial passage of these lymph nodes. Those cells were more metastatic than their parental line, with 30\% developing regional lymph nodes metastasis, and $20 \%$, lung metastasis. By the same method, the $686 \mathrm{LN}$ cell line was injected into the submandibular to mylohyoid muscle of nude mice and a high metastatic cell line (686LN-M2) with a metastasis rate of $50 \%$ was established. ${ }^{92}$ Chen et $\mathrm{al}^{93}$ injected murine $\mathrm{SCC}$ cell line (Pam) into BALB/c SCID mice, forming tumors. They then separated tumor cells, injected them into immunocompetent $\mathrm{BABL} / \mathrm{c}$ mice, resulting in tumor and lymph node metastasis. Cell line selected from the lymph node exhibiting a stronger metastatic ability was named as Pam-LY.

\section{Genetically engineered model}

In 1984, transgenic mouse model was originally utilized in mammary adenocarcinomas and brain tumors studies by Stewart et al ${ }^{94}$ and Brinster et al..$^{95}$ Transgenic and knockout technology can upregulate or downregulate the expression of certain genes, even silence it. So, we can evaluate the effect of the target gene in the origination and progression of HNSCC in immunocompetent environment.

Ras as an oncogene, frequently activated by point mutations or overexpression, plays a crucial role in human tumors. It has been widely used to establish novel mouse models of HNSCC. In 1987, Sinn et al ${ }^{96}$ mated two separate strains of transgenic mice that carry the v-Ha-ras and c-myc, respectively, which were both driven by the mouse mammary tumor virus (MMTV) promoter/enhancer to yield hybrid mice, and resulted in a dramatic and synergistic acceleration of mammary and salivary tumor formation. A similar model has been employed extensively because of its high susceptibility to the development of mammary and salivary tumors. ${ }^{96-101}$ Crossing v-Ha-ras gene (+) FVB/n mice with HPV16 E6/E7(+) C57BL/6 mice, Schreiber et al ${ }^{102}$ observed a remarkable synergy between these two oncogenes in the development of primary mouth, eye, and ear tumors in mice. Using K5-rTA, tet-ras, and tetO-LacZ transgenic mice, Raimondi et $\mathrm{l}^{103}$ proved that inducing expression of $\mathrm{K}$-ras gene under the control of $\mathrm{K} 5$ promoter could trigger a full carcinogenesis. In addition, they successfully established an oral-specific K-ras and p53 two-hit carcinogenesis model system. ${ }^{104}$ Using a similar model, Van der Weyden et a ${ }^{105}$ demonstrated that the activated oncogenic K-ras in the mouse oral mucosa could induce the formation of OSCC. To elucidate the effect of ras mutations in oral cancer formation, Caulin et a ${ }^{106}$ created a novel model that allowed the focal activation of K-ras in stratified epithelia. In a H-ras mutation model, male CB6F1-Tg-rasH2 @Jcl mice, Miyamoto et al ${ }^{107}$ demonstrated that 4-NQO more easily induced tongue and esophageal carcinogenesis.

The p53 protein plays a pivotal role in inducing cell cycle arrest or apoptosis in the presence of DNA damage. A series of transgenic mouse model systems has been created for the sake of understanding the role and mechanism of p53 in the HNSCC occurrence and development. A type of dominant-negative p53 transgenic mouse was shown to be highly susceptible to 4-NQO with higher prevalence and more rapid development of OSCC. ${ }^{108}$ Moral et a ${ }^{109}$ had produced a mouse model that combined constitutive Akt activity (myrAkt) with tumor repressor protein 53 (Trp53) loss and found that it was prone to develop HNSCC. In xeroderma pigmentosum group A gene-deficient $\left(\mathrm{XPA}^{--}\right)$mouse strain carrying mutant alleles for $\mathrm{p} 53$, treated with 4-NQO, Ide et $\mathrm{al}^{110}$ found the accelerated tongue tumor growth might be a consequence of haplonin sufficiency rather than the mutation of $\mathrm{p} 53$ in the context of nucleotide excision repair deficiency. In order to understand the role of p53 in the responses of chemotherapy, Bearss et al and Petit et al ${ }^{100,101}$ used MMTV-ras transgenic mice to interbreed to p 53 knockout mice, and then created a model. In a p53 loss with c-neu overexpression mice model, Brodie et $\mathrm{al}^{111}$ have observed the increased rate of parotid gland tumor onset and no effects on breast tumor formation.

Transforming growth factor-beta (TGF- $\beta$ ), a key regulator of epithelial cell proliferation, immune function, and angiogenesis, is widely studied in human tumors, including HNSCC. ${ }^{112}$ Hyperproliferation, reduced apoptosis, and increased genomic instability are related to defective TGF- $\beta$ signal in epithelial cells. ${ }^{113,114}$ In order to define the role of TGF- $\beta$ RII in HNSCC, Liu et $\mathrm{al}^{115}$ established a 
TGF-ßRII-deleted mouse model. Activation of either K-ras or H-ras in combination with TGF- $\beta$ RII deletion from mouse head-and-neck epithelia caused HNSCC with complete penetrance, some of which progressed to metastases. With the same model system, their further findings demonstrated that the abrogation of TGF- $\beta$ signaling would continuously stimulate $\mathrm{NF}-\kappa \mathrm{B}$, and then result in malignant progression. ${ }^{116}$ Bian et $\mathrm{al}^{117}$ developed an inducible mouse model by knocking out Tgf- $\beta R 1$ and Pten. In this model, NF- $\kappa B$ pathway activation, myeloid-derived suppressor cell infiltration angiogenesis, and immune suppression in the tumor microenvironment were considered to contribute to head and neck carcinogenesis, which indicated that the tumor microenvironment played important role in the HNSCC. ${ }^{117}$ In the same mouse model, by inhibiting mTOR signal, chemopreventive rapamycin treatment significantly delayed the onset of the HNSCC and prolonged survival of mice. ${ }^{118,119}$ PI3K-mTOR inhibition can enhance TP53/p73 expression and significantly inhibit tumor growth alone. ${ }^{120}$ In summary, mouse models with TGF- $\beta$ signaling pathway may be quite useful for the research of HNSCC treatment.

High-risk human papillomaviruses (HPVs) are linked etiologically to a subset of HNSCC. ${ }^{121}$ Jabbar et al ${ }^{122}$ established a model for HPV-associated HNSCC in which they treated transgenic mice expressing the papilloma viral oncoproteins with 4-NQO. They found that the HPV-16 E7 oncoprotein was highly potent at causing HNSCC. They further discovered a striking synergy between E6 and E7 in causing HNSCC. ${ }^{122}$ To study the biological activities of the HPV $16 E 6$ and E7 genes in epithelial cells in vivo, transgenic mice with HPV 16 E6 and E7 were established and tumorigenicity by HPV 16 E6 and E7 was obtained. ${ }^{123,124}$ Further, Song et al ${ }^{125}$ have used transgenic mice that express HPV 16 E6 or E7 in the epidermis to find the mechanism by which HPV 16 E6 or E7 abrogates radiation-induced DNA damage responses in vivo through p53-dependent and p53-independent pathways. ${ }^{125}$ Nguyen et al ${ }^{126}$ generated transgenic mice, K14E6 (Delta 146-151), that express stratified squamous epithelia from the human keratin 14 (K14) promoter. These mice exhibit a radiation response similar to that of the K14E6 (wild-type [WT]) mice, demonstrating that HPV16 E6 retains an ability to inactivate p53. ${ }^{126-128}$ And in this model, E6 has been showed to primarily contribute to the late stages of carcinogenesis that lead to malignancy. ${ }^{129,130}$

Other cancer associated genes, such as $C O X-2, M M P-8$, $X P A$, cyclin D1, and PLAG1, have been utilized to establish models. Zinc-deficient mice with genetic COX-2 deletion developed significantly greater upper aerodigestive tract tumor than WT controls, and presented strong LTA(4)H immunostaining in induced tumors. ${ }^{131}$ The data from MMP-8 knockout mice model system implied that MMP-8 was a protective factor in the growth of mobile tongue SCC. ${ }^{132}$ By assessing the response of $\mathrm{XPA}^{-/}$mice to 4-NQO, the nucleotide excision repair gene $X P A$ was considered as a defensive factor against this carcinogen in vivo. ${ }^{133}$ Cyclin D1 overexpression mice were crossed with p53-deficient mice, then a well-defined model of oral-esophageal cancer was successfully established. It could be a platform for genome-wide analysis, which was found to be useful to test the chemopreventive, diagnostic, and therapeutic strategies. ${ }^{134}$ In PLAG1 transgenic mice, Declercq et $\mathrm{al}^{135}$ found that pleomorphic adenomas of the salivary glands were induced and that both Igf and Wnt signaling pathway were involved.

Experiments in vivo with genetically engineered models have greatly advanced the understanding of tumorigenesis because of interventions at gene level. However, the transgenic model is still not a perfect model. Gene mutations are present in all the cells of transgenic animal since embryogenesis, whereas mutations only occur in few cells surrounded by normal cells rather than generalized cells in humans. This promotes the building of the targeted and inducible models on which gene mutation in the targeted cells of animals can be generated. Andl et al ${ }^{136}$ developed two mouse models targeting ablation of $C d h 1$ and $T g f b r 2$ genes constitutively or inducibly in the oral-esophageal epithelium, which had long latency and could phenocopy the human disease quite accurately. Opitz et al ${ }^{137}$ generated L2-cyclin D1 (L2D1(+)) mice and crossbred these with p53-deficient mice; L2D1(+)/ p53(+/-) mice provided a well-defined, novel, and faithful model of oral-esophageal cancer. ${ }^{137}$ Stairs et al ${ }^{138}$ have generated a conditional knockout model of p120ctn, whereby mice develop preneoplastic and neoplastic lesions in the oral cavity, esophagus, and squamous forestomach. The targeted and inducible models can avoid gene mutation of animal cells, and this model should be widely popularized in the future research of HNSCC.

\section{Conclusion and perspectives}

Mouse models play an important role in HNSCC by providing a model to identify the markers for early diagnosis, understanding the mechanism of tumor initiation and development, and studying the prevention and treatment of HNSCC. There are many different model systems for HNSCC; however, the results obtained from different models may be quite different and dissatisfying to some extent. A real ideal model, which can phenocopy HNSCC progression perfectly, has not been 
established so far. Each model system has its advantages and disadvantages. We cannot use one key to open all the doors, just as one mouse model cannot be used to study all cancers. Cocarcinogenesis may have a better strategy, and this means using more than one method in one mouse model system in order to minimize the disadvantages and make the model more effective. With the development of technology and the enrichment of knowledge and understanding, more perfect HNSCC mouse models could be hopefully established to improve research on HNSCC and help to better treatment eventually.

\section{Acknowledgments}

This work was supported by National Natural Science Foundation of China grants (numbers 81361120399, 81321002, 81272961, 81372891, and 81572650), Fundamental Research Funds of the Central Universities of China (2015), and by State Key Laboratory of Oral Diseases Special Funded Projects (SKLOD201512).

\section{Disclosure}

The authors report no conflicts of interest in this work.

\section{References}

1. Haddad RI, Shin DM. Recent advances in head and neck cancer. N Engl J Med. 2008;359(11):1143-1154.

2. Jemal A, Siegel R, Xu J, et al. Cancer statistics, 2010. CA Cancer J Clin. 2010;60(5):277-300.

3. Jung AC, Ray AM, Ramolu L, et al. Caveolin-1-negative head and neck squamous cell carcinoma primary tumors display increased epithelial to mesenchymal transition and prometastatic properties. Oncotarget. Epub October 12, 2015.

4. Leemans RL, Braakhuis BJ, Brakenhoff RH. The molecular biology of head and neck cancer. Nat Rev Cancer. 2011;11(1):9-22.

5. Caligiuri I, Rizzolio F, Boffo S, et al. Critical choices for modeling breast cancer in transgenic mouse models. J Cell Physiol. 2012;227(8): 2988-2991.

6. Herschkowitz JI, Lubet R. Mouse models of triple negative [basal-like/ claudin low] breast cancer. Breast Dis. 2010;32(1-2):63-71.

7. Cheon DJ, Orsulic S. Mouse models of cancer. Annu Rev Pathol. 2011; 6:95-119.

8. Smith LP, Thomas GR. Animal models for the study of squamous cell carcinoma of the upper aerodigestive tract: a historical perspective with review of their utility and limitations. Part A. Chemically-induced de novo cancer, syngeneic animal models of HNSCC, animal models of transplanted xenogeneic human tumors. Int J Cancer. 2006;118(9): 2111-2122.

9. Lu SL, Herrington H, Wang XJ. Mouse models for human head and neck squamous cell carcinomas. Head Neck. 2006;28(10):945-954.

10. Close DM, Xu T, Sayler GS, et al. In vivo bioluminescent imaging (BLI): noninvasive visualization and interrogation of biological processes in living animals. Sensors (Basel). 2011;11(1):180-206.

11. Bissell MJ, Hines WC. Why don't we get more cancer? A proposed role of the microenvironment in restraining cancer progression. Nat Med. 2011;17(3):320-329.

12. Lorusso G, Ruegg C. The tumor microenvironment and its contribution to tumor evolution toward metastasis. Histochem Cell Biol. 2008; 130(6):1091-1103.
13. Mantovani A, Allavena P, Sica A, et al. Cancer-related inflammation. Nature. 2008;454(7203):436-444.

14. Balkwill F, Mantovani A. Inflammation and cancer: back to Virchow? Lancet. 2001;357(9255):539-545.

15. Hanahan D, Weinberg RA. The hallmarks of cancer. Cell. 2000; 100(1):57-70.

16. Hanahan D, Weinberg RA. Hallmarks of cancer: the next generation. Cell. 2011;144(5):646-674.

17. Flanagan SP. 'Nude', a new hairless gene with pleiotropic effects in the mouse. Genet Res. 1966;8(3):295-309.

18. Pantelouris EM, Hair J. Thymus dysgenesis in nude (nu nu) mice. J Embryol Exp Morphol. 1970;24(3):615-623.

19. Rygaard J, Povlsen CO. Heterotransplantation of a human malignant tumour to "Nude" mice. Acta Pathol Microbiol Scand. 1969;77(4): 758-760.

20. Bosma GC, Custer RP, Bosma MJ. A severe combined immunodeficiency mutation in the mouse. Nature. 1983;301(5900):527-530.

21. Prochazka M, Gaskins HR, Shultz LD, et al. The nonobese diabetic scid mouse: model for spontaneous thymomagenesis associated with immunodeficiency. Proc Natl Acad Sci U S A. 1992;89(8): 3290-3294

22. Shultz LD, Schweitzer PA, Christianson SW, et al. Multiple defects in innate and adaptive immunologic function in NOD/LtSz-scid mice. J Immunol. 1995;154(1):180-191.

23. Povlsen CO, Rygaard J. Heterotransplantation of human epidermoid carcinomas to the mouse mutant nude. Acta Pathol Microbiol Scand A. 1972;80(6):713-717.

24. Braakhuis BJ, Sneeuwloper G, Snow GB. The potential of the nude mouse xenograft model for the study of head and neck cancer. Arch Otorhinolaryngol. 1984;239(1):69-79.

25. Yu FS, Yang JS, Yu CS, et al. Safrole induces apoptosis in human oral cancer HSC-3 cells. J Dent Res. 2011;90(2):168-174.

26. Shen LC, Chen YK, Lin LM, et al. Anti-invasion and anti-tumor growth effect of doxycycline treatment for human oral squamous-cell carcinomain vitro and in vivo studies. Oral Oncol. 2010;46(3):178-184.

27. Ito S, Ozawa S, Ikoma T, et al. Expression of a chemokine BRAK/ CXCL14 in oral floor carcinoma cells reduces the settlement rate of the cells and suppresses their proliferation in vivo. Biomed Res. 2010;31(3): 199-206.

28. Liang X, Zhou H, Liu X, et al. Effect of local hyperthermia on lymphangiogenic factors VEGF-C and -D in a nude mouse xenograft model of tongue squamous cell carcinoma. Oral Oncol. 2010;46(2):111-115.

29. Zhu GQ, Tang YL, Li L, et al. Hypoxia inducible factor $1 \alpha$ and hypoxia inducible factor $2 \alpha$ play distinct and functionally overlapping roles in oral squamous cell carcinoma. Clin Cancer Res. 2010;16(19): $4732-4741$.

30. Harada K, Itashiki Y, Takenawa T, et al. Effects of lentinan alone and in combination with fluoropyrimidine anticancer agent on growth of human oral squamous cell carcinoma in vitro and in vivo. Int J Oncol. 2010;37(3):623-631.

31. Epperly MW, Lai SY, Kanai AJ, et al. Effectiveness of combined modality radiotherapy of orthotopic human squamous cell carcinomas in $\mathrm{Nu} / \mathrm{Nu}$ mice using cetuximab, tirapazamine and MnSOD-plasmid liposome gene therapy. In Vivo. 2010;24(1):1-8.

32. Ekshyyan O, Rong Y, Rong X, et al. Comparison of radiosensitizing effects of the mammalian target ofrapamycin inhibitor CCI-779 to cisplatin in experimental models of head and neck squamous cell carcinoma. Mol Cancer Ther. 2009;8(8):2255-2265.

33. Dinesman A, Haughey B, Gates GA, et al. Development of a new in vivo model for head and neck cancer. Otolaryngol Head Neck Surg. 1990;103(5 Pt 1):766-774.

34. Kawashiri S, Kumagai S, Kojima K, et al. Development of a new invasion and metastasis model of human oral squamous cell carcinomas. Eur J Cancer B Oral Oncol. 1995;31B(4):216-221.

35. Kawashiri S, Kojima K, Kumagai S, et al. Effects of chemotherapy on invasion and metastasis of oral cavity cancer in mice. Head Neck. 2001;23(9):764-771. 
36. Kawashiri S, Noguchi N, Tanaka A, et al. Inhibitory effect of neoadjuvant chemotherapy on metastasis of oral squamous cell carcinoma in a mouse model. Oral Oncol. 2009;45(9):794-797.

37. Umeda M, Yokoo S, Komori T, et al. Experimental model of invasion and metastasis by orthotopic transplantation of oral squamous and adenoid cystic carcinomas into the tongue of nude mice. $\mathrm{Br} \mathrm{J} \mathrm{Oral}$ Maxillofac Surg. 2001;39(5):376-380.

38. Simon C, Nemechek AJ, Boyd D, et al. An orthotopic floor-of-mouth cancer model allows quantification of tumor invasion. Laryngoscope. 1998;108(11 Pt 1):1686-1691.

39. Fang L, Wang H, Zhou L, et al. FOXO3a reactivation mediates the synergistic cytotoxic effects of rapamycin and cisplatin in oral squamous cell carcinoma cells. Toxicol Appl Pharmacol. 2011;251(1):8-15.

40. Yadav A, Kumar B, Datta J, et al. IL-6 promotes head and neck tumor metastasis by inducing epithelial-mesenchymal transition via the JAK-STAT3-SNAIL signaling pathway. Mol Cancer Res. 2011; 9(12):1658-1667.

41. Shinriki S, Jono H, Ota K, et al. Humanized anti-interleukin-6 receptor antibody suppresses tumor angiogenesis and in vivo growth of human oral squamous cell carcinoma. Clin Cancer Res. 2009;15(17): 5426-5434.

42. Jung HM, Phillips BL, Patel RS, et al. Keratinization-associated miR-7 and miR-21 regulate tumor suppressor reversion-inducing cysteinerich protein with kazal motifs (RECK) in oral cancer. J Biol Chem 2012;287(35):29261-29272.

43. Yang $\mathrm{MH}$, Lin BR, Chang $\mathrm{CH}$, et al. Connective tissue growth factor modulates oral squamous cell carcinoma invasion by activating a miR504/FOXP1 signalling. Oncogene. 2012;31(19):2401-2411.

44. Lin YC, Chen HW, Kuo YC, et al. Therapeutic efficacy evaluation of curcumin on human oral squamous cell carcinoma xenograft using multimodalities of molecular imaging. Am J Chin Med. 2010; 38(2):343-358.

45. Patel V, Marsh CA, Dorsam RT, et al. Decreased lymphangiogenesis and lymph node metastasis by mTOR inhibition in head and neck cancer. Cancer Res. 2011;71(22):7103-7112.

46. Frank NY, Schatton T, Frank MH. The therapeutic promise of the cancer stem cell concept. J Clin Invest. 2010;120(1):41-50.

47. Visvader JE, Lindeman GJ. Cancer stem cells in solid tumours: accumulating evidence and unresolved questions. Nat Rev Cancer. 2008 8(10):755-768.

48. Prince ME, Sivanandan R, Kaczorowski A, et al. Identification of a subpopulation of cells with cancer stem cell properties in head and neck squamous cell carcinoma. Proc Natl Acad Sci US A. 2007;104(3): 973-978

49. Clay MR, Tabor M, Owen JH, et al. Single-marker identification of head and neck squamous cell carcinoma cancer stem cells with aldehyde dehydrogenase. Head Neck. 2010;32(9):1195-1201.

50. Sun S, Wang Z. Head neck squamous cell carcinoma c-Met cells display cancer stem cell properties and are responsible for cisplatin-resistance and metastasis. Int J Cancer. 2011;129(10):2337-2348.

51. Shrivastava S, Steele R, Sowadski M, et al. Identification of molecular signature of head and neck cancer stem-like cells. Sci Rep. 2015; 5:7819.

52. Sharkey FE, Fogh J. Metastasis of human tumors in athymic nude mice. Int J Cancer. 1979;24(6):733-738.

53. Johnson JI, Decker S, Zaharevitz D, et al. Relationships between drug activity in NCI preclinical in vitro and in vivo models and early clinical trials. Br J Cancer. 2001;84(10):1424-1431.

54. Macchiarini F, Manz MG, Palucka AK, et al. Humanized mice are we there yet? J Exp Med. 2005;202(10):1307-1311.

55. Hiramatsu H, Nishikomori R, Heike T, et al. Complete of reconstitution of human lymphocytes from cord blood CD34+ cells using the NOD/ SCID/gammacnull mice model. Blood. 2003;102(3):873-880.

56. Kambe N, Hiramatsu H, Shimonaka M, et al. Development of both human connective tissue-type and mucosal-type mast cells in mice from hematopoietic stem cells with identical distribution pattern to human body. Blood. 2004;103(3):860-867.
57. Gardner DG. Spontaneous squamous cell carcinomas of the oral region in domestic animals: a review and consideration of their relevance to human research. Oral Dis. 1996;2(2):148-154.

58. Levy BM, Gorlin R, Gottsegen R. A histologic study of the reaction of skin and mucous membrane to a single application of 9,10-dimethyl1,2-benzanthracene. J Dent Res. 1950;29:678-679.

59. Nishioka H, Hishi K, Kyokane K. Human saliva inactivates mutagenicity of carcinogens. Mutat Res.1981;85:323-333.

60. Salley JJ. Experimental carcinogenesis in the cheek pouch of the Syrian hamster. J Dent Res. 1954;33:253-262.

61. Morris AL. Factors influencing experimental carcinogenesis in the hamster cheek pouch. J Dent Res. 1961;40:3-15.

62. MacDonald DG. Comparison of epithelial dysplasia in hamster cheek pouch carcinogenesis and human oral mucosa. J Oral Pathol. 1981;10:186-191.

63. Nauta JM, Roodenburg JL, Nikkels PG, et al. Comparison of epithelial dysplasia - the 4NQO rat palate model and human oral mucosa. Int J Oral Maxillofac Surg. 1995;24(1 Pt 1):53-58.

64. Ciapparelli L, Retief DH, Fatti LP. The effect of zinc on 9,10-dimethyl1,2-benzanthracene (DMBA) induced salivary gland tumours in the albino rat - a preliminary study. S Afr J Med Sci. 1972;37(3): 85-90.

65. Eveson JW. Animal models of intra-oral chemical carcinogenesis: a review. J Oral Pathol. 1981;10(3):129-146.

66. Hawkins BL, Heniford BW, Ackermann DM, et al. 4NQO carcinogenesis: a mouse model of oral cavity squamous cell carcinoma. Head Neck. 1994;16(5):424-432.

67. Tang XH, Knudsen B, Bemis D, et al. Oral cavity and esophageal carcinogenesis modeled in carcinogen-treated mice. Clin Cancer Res. 2004;10(1 Pt 1):301-313.

68. Chang NW, Pei RJ, Tseng HC, et al. Co-treating with arecoline and 4-nitroquinoline 1-oxide to establish a mouse model mimicking oral tumorigenesis. Chem Biol Interact. 2010;183(1):231-237.

69. Boyd NM, Reade PC. Temporal alterations in cytokeratin expression during experimental oral mucosal carcinogenesis. Carcinogenesis. 1991;12(10):1767-1771.

70. Nauta JM, Roodenburg JL, Nikkels PG, et al. Epithelial dysplasia and squamous cell carcinoma of wistar rat palatal mucosa: 4NQO model. Head Neck. 1996;18(5):441-449.

71. Vered M, Yarom N, Dayan D. 4NQO oral carcinogenesis: animal models, molecular markers and future expectations. Oral Oncol. 2005; 41(4):337-339.

72. Liu X, He R, Chen W. A rat model of tongue mucosa squamous cell carcinoma induced by oral administration of 4NQO in drinking water. Zhonghua Kou Qiang Yi Xue Za Zhi. 1999;34(6):354-356.

73. Rich AM, Reade PC. Epithelial-mesenchymal interactions in experimental oral mucosal carcinogenesis. J Oral Pathol Med. 2001;30(7): 389-397.

74. Baba S, Yamada Y, Hatano Y, et al. Global DNA hypomethylation suppresses squamous carcinogenesis in the tongue and esophagus. Cancer Sci. 2009;100(7):1186-1191.

75. Tang XH, Albert M, Scognamiglio T, et al. A DNA methyltransferase inhibitor and all-trans retinoic acid reduce oral cavity carcinogenesis induced by the carcinogen 4-nitroquinoline 1-oxide. Cancer Prev Res (Phila). 2009;2(12):1100-1110.

76. Hasina R, Martin LE, Kasza K, et al. ABT-510 is an effective chemopreventive agent in the mouse 4-nitroquinoline 1-oxide model of oral carcinogenesis. Cancer Prev Res (Phila). 2009;2(4):385-393.

77. Chang NW, Tsai MH, Lin C, et al. Fenofibrate exhibits a high potential to suppress the formation of squamous cell carcinoma in an oral-specific 4-nitroquinoline 1-oxide/arecoline mouse model. Biochim Biophys Acta. 2011;1812(4):558-564.

78. Zhou G, Hasina R, Wroblewski K, et al. Dual inhibition of vascular endothelial growth factor receptor and epidermal growth factor receptor is an effective chemopreventive strategy in the mouse 4-NQO model of oral carcinogenesis. Cancer Prev Res (Phila). 2010;3(11): 1493-1502. 
79. Sheu JJ, Hua CH, Wan L, et al. Functional genomic analysis identified epidermal growth factor receptor activation as the most common genetic event in oral squamous cell carcinoma. Cancer Res. 2009;69(6): 2568-2576.

80. Wong DY, Feinberg SE. Incidence of growth of syngeneic oral squamous cell carcinoma in C57B1 bg/bg beige mice. Zhonghua Yi Xue Za Zhi (Taipei). 1990;45(1):53-59.

81. Fu KK, Rayner PA, Lam KN. Modification of the effects of continuous low dose rate irradiation by concurrent chemotherapy infusion. Int J Radiat Oncol Biol Phys. 1984;10(8):1473-1478.

82. O’Malley BW Jr, Cope KA, Johnson CS, et al. A new immunocompetent murine model for oral cancer. Arch Otolaryngol Head Neck Surg. 1997;123(1):20-24.

83. Hier MP, Black MJ, Shenouda G, et al. A murine model for the immunotherapy of head and neck squamous cell carcinoma. Laryngoscope. 1995;105(10):1077-1080.

84. Thomas GR, Chen Z, Oechsli MN, et al. Decreased expression of CD80 is a marker for increased tumorigenicity in a new murine model of oral squamous-cell carcinoma. Int J Cancer. 1999;82(3):377-384.

85. Thomas GR, Chen Z, Enamorado I, et al. IL-12- and IL-2-induced tumor regression in a new murine model of oral squamous-cell carcinoma is promoted by expression of the CD80 co-stimulatory molecule and interferon-gamma. Int J Cancer. 2000;86(3):368-374.

86. Ahmed SU, Okamoto M, Oshikawa T, et al. Anti-tumor effect of an intratumoral administration of dendritic cells in combination with TS-1, an oral fluoropyrimidine anti-cancer drug, and OK-432, a streptococcal immunopotentiator: involvement of toll-like receptor 4. J Immunother. 2004;27(6):432-441.

87. Cui N, Nomura T, Noma H, et al. Effect of YM529 on a model of mandibular invasion by oral squamous cell carcinoma in mice. Clin Cancer Res. 2005;11(7):2713-2719.

88. Nomura T, Shibahara T, Katakura A, et al. Establishment of a murine model of bone invasion by oral squamous cell carcinoma. Oral Oncol. 2007;43(3):257-262.

89. Lee JK, Lim SC, Kim HD, et al. KITENIN represents a more aggressive phenotype in a murine model of oral cavity squamous carcinoma. Otolaryngol Head Neck Surg. 2010;142(5):747-752.

90. Furuta H, Osawa K, Shin M, et al. Selective inhibition of NF-кB suppresses bone invasion by oral squamous cell carcinoma in vivo. Int J Cancer. 2012;131(5):E625-E635.

91. Myers JN, Holsinger FC, Jasser SA, et al. An orthotopic nude mouse model of oral tongue squamous cell carcinoma. Clin Cancer Res. 2002; $8(1): 293-298$.

92. Zhang X, Liu Y, Gilcrease MZ, et al. A lymph node metastatic mouse model reveals alterations of metastasis-related gene expression in metastatic human oral carcinoma sublines selected from a poorly metastatic parental cell line. Cancer. 2002;95(8):1663-1672.

93. Chen Z, Smith CW, Kiel D, et al. Metastatic variants derived following in vivo tumor progression of an in vitro transformed squamous cell carcinoma line acquire a differential growth advantage requiring tumor-host interaction. Clin Exp Metastasis. 1997;15(5):527-537.

94. Stewart TA, Pattengale PK, Leder P. Spontaneous mammary adenocarcinomas in transgenic mice that carry and express MTV/myc fusion genes. Cell. 1984;38(3):627-637.

95. Brinster RL, Chen HY, Messing A, et al. Transgenic mice harboring SV40 T-antigen genes develop characteristic brain tumors. Cell. 1984;37(2):367-379.

96. Sinn E, Muller W, Pattengale P, et al. Coexpression of MMTV/v-Haras and MMTV/c-myc genes in transgenic mice: synergistic action of oncogenes in vivo. Cell. 1987;49(4):465-475.

97. Adnane J, Jackson RJ, Nicosia SV, et al. Loss of p21WAF1/CIP1 accelerates Ras oncogenesis in a transgenic/knockout mammary cancer model. Oncogene. 2000;19(47):5338-5347.

98. Hundley JE, Koester SK, Troyer DA, et al. Increased tumor proliferation and genomic instability without decreased apoptosis in MMTV-ras mice deficient in p53. Mol Cell Biol. 1997;17(2):723-731.
99. Barrington RE, Subler MA, Rands E, et al. A farnesyltransferase inhibitor induces tumor regression in transgenic mice harboring multiple oncogenic mutations by mediating alterations in both cell cycle control and apoptosis. Mol Cell Biol. 1998;18(1):85-92.

100. Bearss DJ, Subler MA, Hundley JE, et al. Genetic determinants of response to chemotherapy in transgenic mouse mammary and salivary tumors. Oncogene. 2000;19(8):1114-1122.

101. Petit T, Bearss DJ, Troyer DA, et al. p53-independent response to cisplatin and oxaliplatin in MMTV-ras mouse salivary tumors. Mol Cancer Ther. 2003;2(2):165-171.

102. Schreiber K, Cannon RE, Karrison T, et al. Strong synergy between mutant ras and HPV16 E6/E7 in the development of primary tumors. Oncogene. 2004;23(22):3972-3979.

103. Raimondi AR, Vitale-Cross L, Amornphimoltham P, et al. Rapid development of salivary gland carcinomas upon conditional expression of K-ras driven by the cytokeratin 5 promoter. Am J Pathol. 2006;168(5):1654-1665.

104. Raimondi AR, Molinolo A, Gutkind JS. Rapamycin prevents early onset of tumorigenesis in an oral-specific K-ras and p53 two-hit carcinogenesis model. Cancer Res. 2009;69(10):4159-4166.

105. Van der Weyden L, Alcolea MP, Jones PH, et al. Acute sensitivity of the oral mucosa to oncogenic K-ras. J Pathol. 2011;224(1):22-32.

106. Caulin C, Nguyen T, Longley MA, et al. Inducible activation of oncogenic K-ras results in tumor formation in the oral cavity. Cancer Res. 2004;64(15):5054-5058.

107. Miyamoto S, Yasui Y, Kim M, et al. A novel rasH2 mouse carcinogenesis model that is highly susceptible to 4-NQO-induced tongue and esophageal carcinogenesis is useful for preclinical chemoprevention studies. Carcinogenesis. 2008;29(2):418-426.

108. Zhang Z, Wang Y, Yao R, et al. p53 Transgenic mice are highly susceptible to 4-nitroquinoline-1-oxide-induced oral cancer. Mol Cancer Res. 2006;4(6):401-410.

109. Moral M, Segrelles C, Lara MF, et al. Akt activation synergizes with Trp53 loss in oral epithelium to produce a novel mouse model for head and neck squamous cell carcinoma. Cancer Res. 2009;69(3): 1099-1108.

110. Ide F, Kitada M, Sakashita H, et al. p53 haploinsufficiency profoundly accelerates the onset of tongue tumors in mice lacking the xeroderma pigmentosum group A gene. Am J Pathol. 2003;163(5): 1729-1733.

111. Brodie SG, Xu X, Li C, et al. Inactivation of p53 tumor suppressor gene acts synergistically with c-neu oncogene in salivary gland tumorigenesis. Oncogene. 2001;20(12):1445-1454.

112. Park Y, Kim W, Lee JM, et al. Cytoplasmic DRAK1 overexpressed in head and neck cancers inhibits TGF- $\beta 1$ tumor suppressor activity by binding to Smad3 to interrupt its complex formation with Smad4. Oncogene. 2015;34(39):5037-5045.

113. Bragado $P$, Estrada $Y$, Parikh F, et al. TGF- $\beta 2$ dictates disseminated tumour cell fate in target organs through TGF- $\beta$-RIII and $\mathrm{p} 38 \alpha / \beta$ signalling. Nat Cell Biol. 2013;15(11):1351-1361.

114. White RA, Malkoski SP, Wang XJ. TGF $\beta$ signaling in head and neck squamous cell carcinoma. Oncogene. 2010;29(40):5437-5446.

115. Liu L, Tang XH, Scognamiglio T, et al. Oral carcinogenesis induced by 4-nitroquinoline 1-oxide in lecithin:retinol acyltransferase gene knockout mice. J Nutr Biochem. 2010;21(10):975-982.

116. Cohen J, Chen Z, Lu SL, et al. Attenuated transforming growth factor beta signaling promotes nuclear factor-kappaB activation in head and neck cancer. Cancer Res. 2009;69(8):3415-3424.

117. Bian Y, Hall B, Sun ZJ, et al. Loss of TGF-b signaling and PTEN promotes head and neck squamous cell carcinoma through cellular senescence evasion and cancer-related inflammation. Oncogene. 2012; 31(28):3322-3332.

118. Sun ZJ, Zhang L, Hall B, et al. Chemopreventive and chemotherapeutic actions of mTOR inhibitor in genetically defined head and neck squamous cell carcinoma mouse model. Clin Cancer Res. 2012;18(19): 5304-5313. 
119. Sun ZJ, Zhang L, Zhang W, et al. Inhibition of mTOR reduces anal carcinogenesis in transgenic mouse model. PLoS One. 2013;8(10): e74888.

120. Herzog A, Bian Y, Vander Broek R, et al. PI3K/mTOR inhibitor PF-04691502 antitumor activity is enhanced with induction of wildtype TP53 in human xenograft and murine knockout models of head and neck cancer. Clin Cancer Res. 2013;19(14):3808-3819.

121. Li Y, Li B, Xu B, et al. Expression of p53, p21(CIP1/WAF1) and eIF4E in the adjacent tissues of oral squamous cell carcinoma: establishing the molecular boundary and a cancer progression model. Int J Oral Sci. 2015;7:161-168.

122. Jabbar S, Strati K, Shin MK, Pilot HC, Lambert PF. Human papillomavirus type 16 E6 and E7 oncoproteins act synergistically to cause head and neck cancer in mice. Virology. 2010;407(1):60-67.

123. Griep AE, Herber R, Jeon S, et al. Tumorigenicity by human papillomavirus type 16 E6 and E7 in transgenic mice correlates with alterations in epithelial cell growth and differentiation. J Virol. 1993; 67(3):1373-1384

124. Herber R, Liem A, Pitot H, et al. Squamous epithelial hyperplasia and carcinoma in mice transgenic for the human papillomavirus type 16 E7 oncogene. J Virol. 1996;70(3):1873-1881.

125. Song S, Gulliver GA, Lambert PF. Human papillomavirus type 16 E6 and E7 oncogenes abrogate radiation-induced DNA damage responses in vivo through p53-dependent and p53-independent pathways. Proc Natl Acad Sci U S A. 1998;95(5):2290-2295.

126. Nguyen ML, Nguyen MM, Lee D, et al. The PDZ ligand domain of the human papillomavirus type 16 E6 protein is required for E6's induction of epithelial hyperplasia in vivo. J Virol. 2003;77(12):6957-6964.

127. Arbeit JM, Münger K, Howley PM, et al. Progressive squamous epithelial neoplasia in K14-human papillomavirus type 16 transgenic mice. J Virol. 1994;68(7):4358-4368.

128. Ghim S, Jenson AB, Bubier JA, et al. Cataracts in transgenic mice caused by a human papillomavirus type 18 E7 oncogene driven by KRT1-14. Exp Mol Pathol. 2008;85(2):77-82.
129. Song S, Pitot HC, Lambert PF. The human papillomavirus type 16 E6 gene alone is sufficient to induce carcinomas in transgenic animals. J Virol. 1999;73(7):5887-5893.

130. Song S, Liem A, Miller JA, et al. Human papillomavirus types 16 E6 and E7 contribute differently to carcinogenesis. Virology. 2000; 267(2):141-150.

131. Fong LY, Jiang Y, Riley M, et al. Prevention of upper aerodigestive tract cancer in zinc-deficient rodents: inefficacy of genetic or pharmacological disruption of COX-2. Int J Cancer. 2008;122(5): 978-989.

132. Korpi JT, Kervinen V, Mäklin H, et al. Collagenase-2 (matrix metalloproteinase-8) plays a protective role in tongue cancer. Br J Cancer. 2008;98(4):766-775.

133. Ide F, Oda H, Nakatsuru $Y$, et al. Xeroderma pigmentosum group A gene action as a protection factor against 4-nitroquinoline 1-oxide-induced tongue carcinogenesis. Carcinogenesis. 2001;22(4):567-572.

134. Opitz OG, Quante M, von Werder A, et al. A mouse model of oralesophageal carcinogenesis. Onkologie. 2005;28(1):44-48.

135. Declercq J, Van Dyck F, Van Damme B, et al. Upregulation of Igf and Wnt signalling associated genes in pleomorphic adenomas of the salivary glands in PLAG1 transgenic mice. Int J Oncol. 2008;32(5): 1041-1047.

136. Andl T, Le Bras GF, Richards NF, et al. Concerted loss of TGF $\beta$ mediated proliferation control and E-cadherin disrupts epithelial homeostasis and causes oral squamous cell carcinoma. Carcinogenesis. 2014;35(11):2602-2610.

137. Opitz OG, Harada H, Suliman Y, et al. A mouse model of human oral-esophageal cancer. J Clin Invest. 2002;110(6):761-769.

138. Stairs DB, Bayne LJ, Rhoades B, et al. Deletion of p120-catenin results in a tumor microenvironment with inflammation and cancer that establishes it as a tumor suppressor gene. Cancer Cell. 2011 $19(4): 470-483$
OncoTargets and Therapy

\section{Publish your work in this journal}

OncoTargets and Therapy is an international, peer-reviewed, open access journal focusing on the pathological basis of all cancers, potential targets for therapy and treatment protocols employed to improve the management of cancer patients. The journal also focuses on the impact of management programs and new therapeutic agents and protocols on

\section{Dovepress}

patient perspectives such as quality of life, adherence and satisfaction. The manuscript management system is completely online and includes a very quick and fair peer-review system, which is all easy to use. Visit http://www.dovepress.com/testimonials.php to read real quotes from published authors. 